\title{
Entre pozas y presas: el principal sistema de regadío tradicional de Galicia: morfología tradicional, lucha contra la industrialización tardía gallega y regulación comunitaria de su agua.
}

\author{
Benito Márquez Castro \\ Universidade de Vigo \\ bieitomarquescastro@live.com
}

\begin{abstract}
Resumen. Este artículo versa sobre el regadío tradicional en Galicia, surgido debido a la necesidad de agua para los prados y fincas en la época estival. En especial, sobre el sistema de pozas y presas, construcciones surgidas de la necesidad de acumular agua para hacer un uso más eficaz de la misma. Estas construcciones, de las cuales ya se tienen noticias en época medieval, han llegado hasta nuestros días. Sin embargo, su morfología tradicional ha sufrido, sobre todo a partir de mediados del siglo XX debido a la industrialización tardía de Galicia, unos cambios que no han contado con el respeto necesario en tanto, además de bienes funcionales, también son parte de nuestro legado histórico y social. Este será uno de los aspectos sobre los que haremos más incidencia en este artículo. Por último, nos centraremos también en las diferentes formas de regulación del uso de estas construcciones tradicionales que tienen en su mayoría un carácter comunitario.
\end{abstract}

Palabras Clave: Pozas, Presas, Regadío tradicional, Galicia, Regulación.

\begin{abstract}
This article focuses on the Galician traditional irrigation, emerged due to the need for water for meadows and lands in summertime. Specially, on the system of the so-called pozas and presas, structures arising from the need of accumulating water to make a more effective use of it. These structures, of which we know already from medieval times, have survived up to nowadays. However, its traditional morphology has undergone, especially from the mid-twentieth century, due to the Galician late industrialization, some changes that have not had the necessary respect as they are also, as well as functional goods, part of our historic and social legacy. This will be one of the aspects about which we will focus on especially on this article. Finally, we also approach on the different forms of regulation of the use of these traditional structures, which mostly have a communal dimension.
\end{abstract}

Key words: Ponds, Galician Traditional Irrigation, Regulation.

\section{Introducción}

Aunque Galicia se sitúa en la zona húmeda de la Península Ibérica y que la abundancia de agua es considerable, los diferentes prados y fincas han necesitado desde tiempos inmemoriales, sobre todo en los meses estivales, del uso del regadío (las conocidas regas ${ }^{\top}$ ). Esta necesidad dio en Galicia en la creación de diversos sistemas específicos para la acumulación de agua y su posterior dispersión por las diferentes fincas y prados, sistemas tradicionales que en la mayoría de los casos no presentan ni una gran envergadura ni tampoco un funcionamiento técnico complejo. Su dificultad reside, más en que estos aspectos, en su desenvolvimiento como sistemas comunales, donde una misma infraestructura de captación de agua servía -y sirve- a numerosos vecinos de la parroquia, lo que implicó una organización compleja con base, esencialmente, en el derecho consuetudinario.

\footnotetext{
${ }^{1}$ Pondremos, en cursiva, la terminología tradicional relativa al regadío en Galicia, en idioma gallego, por ser el que se utiliza mayoritariamente en la agricultura tradicional y en el que son dichos los diversos términos técnicos al respecto.
} 
De esta necesidad del regadío surgieron diferentes sistemas, nacidos de la adaptación a los diferentes condicionantes que el medio establecía de antemano en las diferentes zonas gallegas. Entre estos condicionantes, destacaban, entre otros, la ubicación de las tierras que se pretendía regar y la ubicación del manantial, y por otro, la cantidad de agua que se podía obtener. Debido a ello, surgieron así cuatro tipologías principales dentro del sistema tradicional gallego de las regas: las lovadas ${ }^{2}$, los pozos pero a la vez divergentes entre sí.

En nuestro estudio tan solo nos centraremos en los dos últimos -las pozas y las presas-, que son los más importantes y los que más presencia han tenido y siguen teniendo hoy en día. El sistema de las pozas y el sistema de las presas, aunque clasificables como dos sistemas aparte, no responden estrictamente a dos sistemas totalmente diferentes en sí, sino más bien a dos estados evolutivos diferentes de un mismo sistema. Este diferente estado evolutivo no parte de ningún condicionante temporal, en el sentido de que las menos evolucionadas sean más antiguas, sino que parte de la diferente adaptación al espacio y a los condicionantes anteriormente señalados, como la mayor o menor cantidad de agua que llevan, su envergadura, etc. Ambos sistemas consisten, grosso modo, en unas construcciones en el terreno en los que se acumula agua proveniente de minas, riachuelos, etc., para posteriormente dispersarla de forma controlada por las fincas y prados.

El sistema menos evolucionado es el de las pozas. Éstas tienen la función de juntar el agua que proviene de un pequeño manantial que suele estar a pocos metros, para que no se pierda por el lugar, siendo de muy pequeña envergadura. Las presas, por el contrario, se dan en lugares con un caudal de agua importante, como riachuelos, teniendo por ello una mayor envergadura, que posibilita su capacidad para regar una gran extensión, y una mayor complejidad técnica y estructural. Es el sistema más extendido por la geografía gallega.

Debido a su envergadura y al hecho de ser usados por muchos vecinos, todas estas presas tienen nombre propio, que sirve a la comunidad como punto georreferencial. Las pozas, en cambio, debido a su menor tamaño e importancia no lo tienen en algunas ocasiones.

\footnotetext{
${ }^{2}$ El sistema de las lovadas o levadas se dio en lugares por donde pasa un río. Se trata de canales de un tamaño considerable, que parten de éstos y que se dirigen hacia las fincas ribereñas, llevando su agua. La abundancia de esta durante todo el año en Galicia hacía que no fuese necesario ningún sistema de almacenamiento, sino que tan solo era necesario guiar el agua por el canal -la lovada- cuando se quisiese (aunque no eran pocas las veces que existieron problemas con los molinos fluviales que se encontraban río abajo, por el desvío de agua que provocaba este sistema). Su infraestructura no difiere prácticamente de aquellas utilizadas por los molinos que reciben su mismo nombre. Sobre un ejemplo de las lovadas en el sur de Pontevedra, cfr. Vázquez Martínez, A. (1946-1947): Las 'levadas’ de Arbo, El Museo de Pontevedra, 4;172-185; Para más información sobre las lovadas de los molinos, véase Caamaño Suárez, M. (2003): As construccións da arquitectura popular: Patrimonio etnográfico de Galicia, Santiago de Compostela, Consello Galego de Colexios de Aparelladores e Arquitectos Técnicos, pp. 344-360.

${ }^{3} \mathrm{El}$ sistema de los pozos es la tipología más básica y simple, siendo pequeñas construcciones para un uso del agua restringido a un particular -también los hay comunales, aunque en mucha menor medida-, para regar normalmente una única finca o prado en el que se encontraba ubicado el mismo. Esta es una de las soluciones que se utilizaba sobre todo en aquellos prados donde el agua brotaba prácticamente a ras del suelo y donde no había que ahondar mucho en el terreno para obtener un manantial suficiente, como los lameiros (barrales). Estos pozos suelen tener forma circular y a veces estaban encintados con piedras, cuando el terreno era menos firme. Debido a que esta es una solución para un particular solo, es la menos frecuente en el agro gallego.
} 

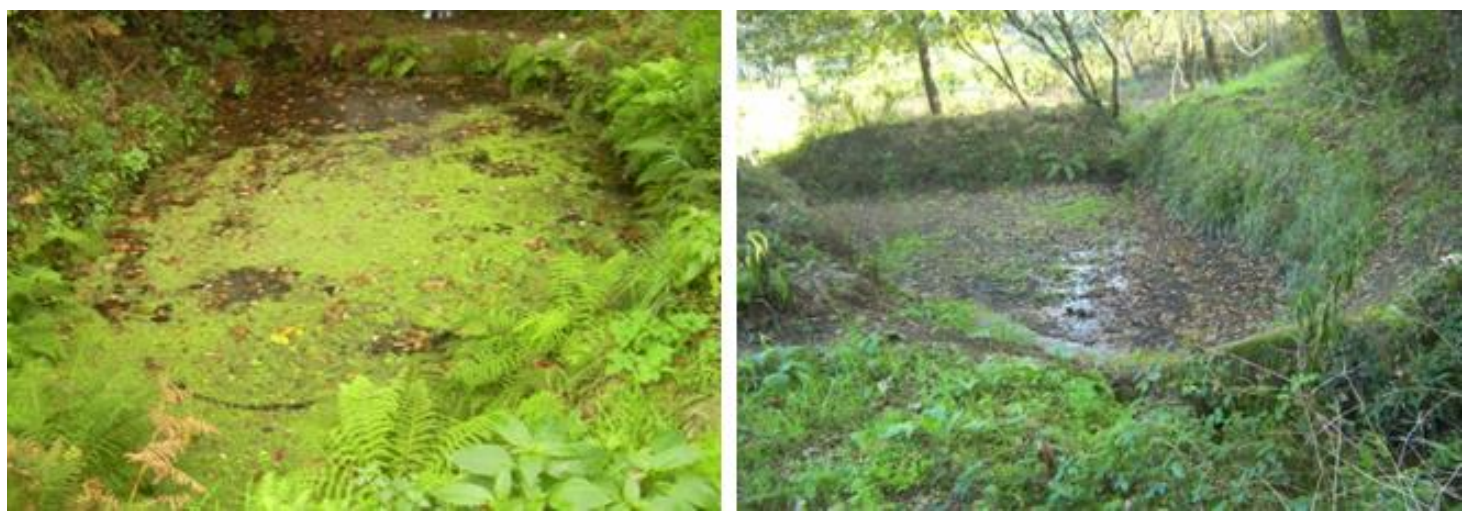

Fig. 1. Ejemplo de poza y presa, respectivamente, donde se pueden apreciar sus características y morfología tradicionales.

Del nombre que reciben estas pozas y presas (sobre todo de éstas últimas) surge a veces también el nombre de los riachuelos. Asimismo, a veces, cuando se da la existencia de varias de estas construcciones en uno de ellos, los campesinos le dan a cada parte del riachuelo próximo a la presa el nombre de esta. Es decir, un mismo riachuelo recibe diferentes nombres en su recorrido, viniéndole dado cada uno de ellos por la presa más próxima. Las parecidas semejanzas existentes entre estos dos sistemas llevaron a que las gentes del rural hablasen indistintamente de presas o pozas para referirse a estas dos últimas tipologías, produciéndose muchas veces entre estos dos términos un caso de sinonimia. Esto no quiere decir que no distingan las diferentes tipologías, sino que tan solo no identifican estos nombres con una tipología concreta. Así, sucede que en algunos lugares el término poza ganó en significado y abarca las dos tipologías, olvidándose el término de presa, que no es utilizado; y viceversa, en otras parroquias se utiliza el término presa, y no el de poza, que ha sido suplantado. Cuando este término de presa gana en significado y abarca las dos tipologías, para diferenciar una de la otra surgen palabras derivadas de ellas (diminutivos casi siempre), como presón o presiña, cuando en realidad están hablando de pozas; y al revés, cuando es el término poza el que gana en significado, este nombre se reserva para las tradicionales presas, y para las tradicionales pozas, tipológicamente hablando, utilizan el término pozanco.

\section{De la ubicación de las pozas y presas}

Las pozas eran construcciones destinadas a albergar la poca agua que brotaba de algún pequeño manantial, con el fin de que esta se concentrase en un lugar único, para después poder, de forma controlada, dispersarla por las fincas y prados, que no solían ser muchos. Normalmente, donde hay una poza, también existe una fuente (a veces también sucede lo mismo en las presas, pero es aquí es ya algo testimonial), que recibe el mismo nombre de ésta y cuya agua no se pierde o desparrama al caer, sino que es conducida hacia estos lugares de acumulación. En definitiva, estamos delante de una de las características básicas de la sociedad rural gallega: el aprovechamiento máximo de todos los recursos que existen a su alrededor.

La construcción de las presas se llevó a cabo en los riachuelos, principalmente inseridas en su propio lecho natural y utilizando alguno de los terraplenes de los pequeños valles que forman como paredes, siendo usual la existencia de diversas construcciones de este tipo en 
el seno de estos canales naturales, una detrás de otra, a una distancia prudencial que no permita que se entorpezcan entre ellas (aunque a veces sucede) y que una le quite agua a la otra. Esto implicaba, pues, que todas ellas estaban comunicadas entre sí, y que parte de la regulación de las aguas se hiciese en común entre los regantes de las distintas presas, con el objetivo de que los de la primera no cortasen el curso de agua a cualquier hora impidiendo que los de la segunda pudiesen llenar la suya.

En otras ocasiones, aunque en menor medida, las presas no se construían en el propio lecho del riachuelo, sino cerca de este. Aprovechando normalmente una curva del propio lecho, para aprovechar la fuerza e inercia del agua, tapaban el riachuelo, y guiaban el agua por un canal construido ex profeso por los campesinos para cambiar el curso natural y hacer que este pasase por la presa. Si el riachuelo no pasaba por el mejor sitio para regar las fincas, se hacía que pasase.

\section{De la morfología y características estructurales de las pozas y presas tradicionales}

Como dijimos anteriormente, las pozas y las presas pueden ser consideradas un mismo sistema de regadío tradicional en diferente estado evolutivo. Esto puede verse fácilmente al analizar la morfología y las características estructurales prototípicas de estos dos tipos de construcciones destinadas a la acumulación de agua.

Las pozas, como dijimos anteriormente, son las menos evolucionadas y las de menor envergadura. Normalmente presentan una forma circular u ovalada, y se encuentran un nivel por debajo del terreno natural. La salida del agua normalmente se hace a través de un pequeño canal de regadío (rego), que se tapa y destapa dependiendo de si el campesino quiere acumular el agua o regar, con terrones y algunas piedras. Durante la época de lluvias, la poza se llena de maleza, que los campesinos limpian justo antes de la llegada de la época estival. En este momento, esta maleza, así como restos de arena y tierra traídos por el agua, se depositan alrededor de la poza, en un acto cíclico por el que se va arreglando cualquier desperfecto de la construcción, autoregenerando ésta.

Una vez que estas construcciones crecen en tamaño debido a la gran cantidad de agua que se va a acumular, crece también la dificultad técnica con que son construidas. De las simples pozas que reciben el agua de una pequeña mina, manantial o de una fuente, pasamos a las presas en riachuelos, donde la cantidad de agua es inmensamente superior. De ello, surgen nuevos problemas y por tanto nuevas soluciones, que suponen la aplicación de ciertos avances y mejoras técnicas en lo que serían estas pozas de riachuelo. Podemos afirmar, en definitiva, que las presas son simples pozas que, debido a la gran cantidad de agua con la que tenían que lidiar, sufrieron una serie de sofisticaciones para solucionar los problemas que esto planteaba.

La forma de éstas presas nos viene dada por la ubicación mayoritaria de éstas en el propio lecho de los riachuelos, dando por resultado una forma triangular, siendo el vértice superior del triángulo la entrada del agua (y del riachuelo, por tanto) y estando la salida en alguna parte de la base (difiere esto dependiendo del diferente curso que siga el riachuelo, aunque normalmente se encontraba en el medio de la base). Esta forma triangular varía también de unos sitios a otros, dándose presas en forma de triángulo equilátero o a veces isósceles, cuando el caudal es mayor, alargándose para albergar más cantidad de agua. 
Sus paredes, al igual que en las pozas, están hechas con tierra, pero aquí aprovechan normalmente, para alguno de los lados, parte del terraplén del propio lecho del riachuelo, en forma de U. Cuando esto sucede, a veces éste se empedraba con pequeños piedras informes (cachotes), con el fin último de ayudar a la sujeción del terraplén y que no se fuese viniendo abajo por el agua.

Estos muros de tierra eran anualmente acondicionados. Antes de la época del regadío, todos los regantes se juntaban y limpiaban la presa, que durante todo el año veía como le iba creciendo maleza y como se llenaban de tierra y barro, que el agua había estado arrastrando consigo. Todo el material extraído de esta limpieza era tirado encima de las propias paredes de tierra de la presa, mejorando los posibles desperfectos y acondicionándolas, al igual que se hacía, pero con mucho menor importancia y prácticamente testimonial, en las pozas. Estamos, pues, delante de una continua remodelación de estas paredes, año a año y de una cíclica autorreconstrucción.

Al igual que las pozas, estas construcciones están un nivel por debajo del terreno natural, lo que implica que en algún caso, cuando la hondura de la presa es considerable y no se podía entrar fácilmente, se colocaban unos postes de granito enclavados en uno de los muros de la construcción, para poder bajar dentro de la misma con facilidad, para taparla, destaparla, para limpiarla, etc. La altura de estos muros es variable, dependiendo de factores diversos, como la ubicación, el caudal o la cantidad de fincas y prados a regar. Normalmente tienen sobre un metro de altura, a veces más y otras veces menos.

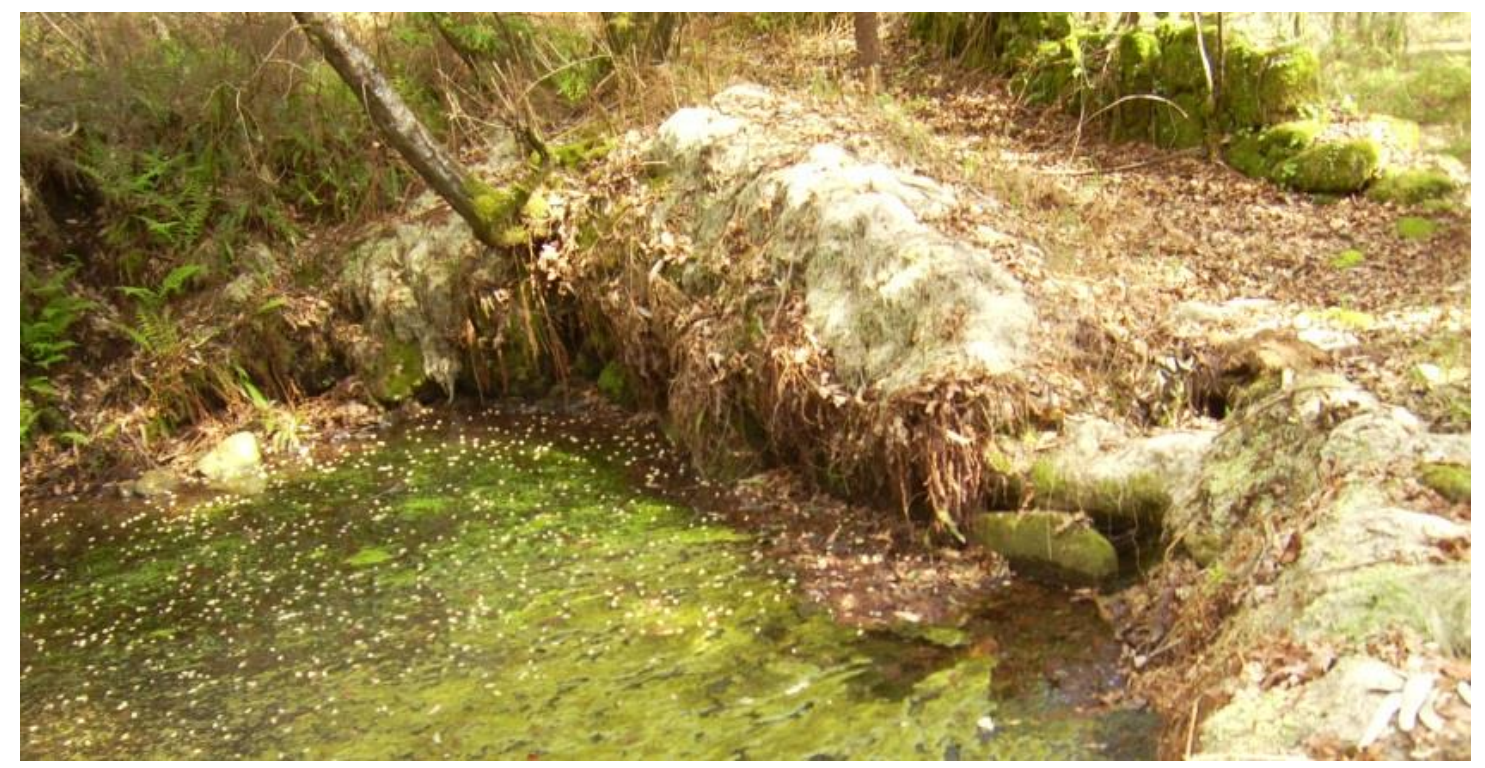

Fig. 2. Ejemplo de presa tradicional donde se pueden observar los restos de la limpieza de la misma depositados alrededor de sí, encima de las paredes, como método tradicional de autorregeneración y acondicionamiento de la construcción.

La necesidad de controlar la salida de agua tanto de las pozas como de las presas trajo consigo una solución técnica cuya complejidad va pareja a la monumentalidad de la presa. Así, en las pozas más pequeñas, este problema se solucionaba con unos cuantos terrones y algunas piedras. La poca fuerza del agua hacía innecesario una complejidad mayor. Las pozas más grandes comenzaron a utilizar más piedras y menos terrones, puesto que la resistencia de las primeras es considerablemente mayor. En las presas, es decir, en construcciones que 
debía soportar una presión del agua mayor, esta necesidad de tapar la salida del agua se mostraba imposible con terrones y piedras. Así pues, surgió una estructura pétrea, el boceiro o buceiro, también llamado boca (la etimología de la palabra boceiro surge de ésta otra). Se trata, simplemente, de varias piedras colocadas de tal forma que dejaban un hueco de forma cuadrangular. El tamaño de este hueco es variable, dependiendo de cada construcción, pero normalmente era grande, ya que era necesario que durante el invierno y durante la época de lluvia, cuando el caudal subiese fuertemente, la construcción permitiese una salida de agua tranquila y constante, para que no bordase por encima de la presa y que no se hiciese un tapón que destruyese la construcción. En la época contraria, es decir, en la época estival de regadío, los campesinos necesitaban lo contrario, es decir, tapar fácilmente este gran hueco del buceiro. La imposibilidad de hacerlo con terrones y piedras trajo consigo la aparición de las llamadas pedras do buceiro. Se trataba de una piedra de granito, labrada y muy cuidada, de forma cuadrangular, con los lados redondeados, y en uno de estos, el que sería la base, un pequeño rebaje en forma de semicírculo pequeño. Esta piedra, verdadera obra de arte rural, era mínimamente más pequeña que el agujero del buceiro, en tanto en cuanto debía encajar en ese hueco. Su función era la siguiente: a la hora de tapar la salida del agua, el buceiro, se colocaba en el hueco esta piedra, con el rebaje hacia abajo, tapando la mayor parte de este, se encintaba con un poco de barro (ya que nunca encajaban a la perfección) y se echaba terrones sobre ese rebaje inferior. Era mucho más fácil tapar un pequeño agujero que toda la boca, y esta piedra, de dimensiones acordes a la presa, aguantaba perfectamente la presión y el envite del agua. Esta piedra se colocaba el primer día de regadío, y no se quitaba hasta el último, en que se retiraba para que el agua saliese de forma natural.

Así, para regar, tan solo era necesario tapar y destapar el pequeño rebaje. Esto se hacía desde encima de la presa, con un simple palo largo, removiendo un poco los terrones que se habían colocado sobre este pequeño agujero que, con la propia fuerza del agua y removiendo un poco la zona, se eliminaban los terrones y comenzaba a correr el agua por el cauce que previamente habían marcados los campesinos.

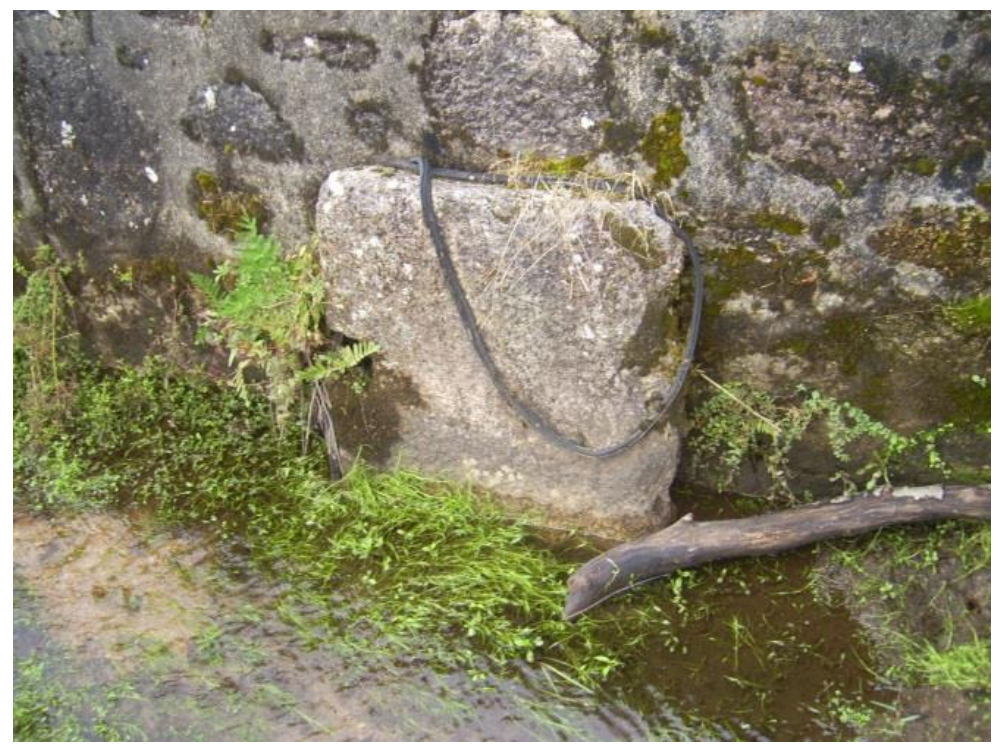

Fig. 3. Pedra de buceiro de grandes dimensiones, actualmente en desuso, apoyada en uno de las paredes de una presa. La sustitución del mecanismo tradicional del buceiro por mecanismos de apertura y cierre modernos han provocado la inutilización de estas piedras labradas, provocando su progresivo abandono y destrucción. 


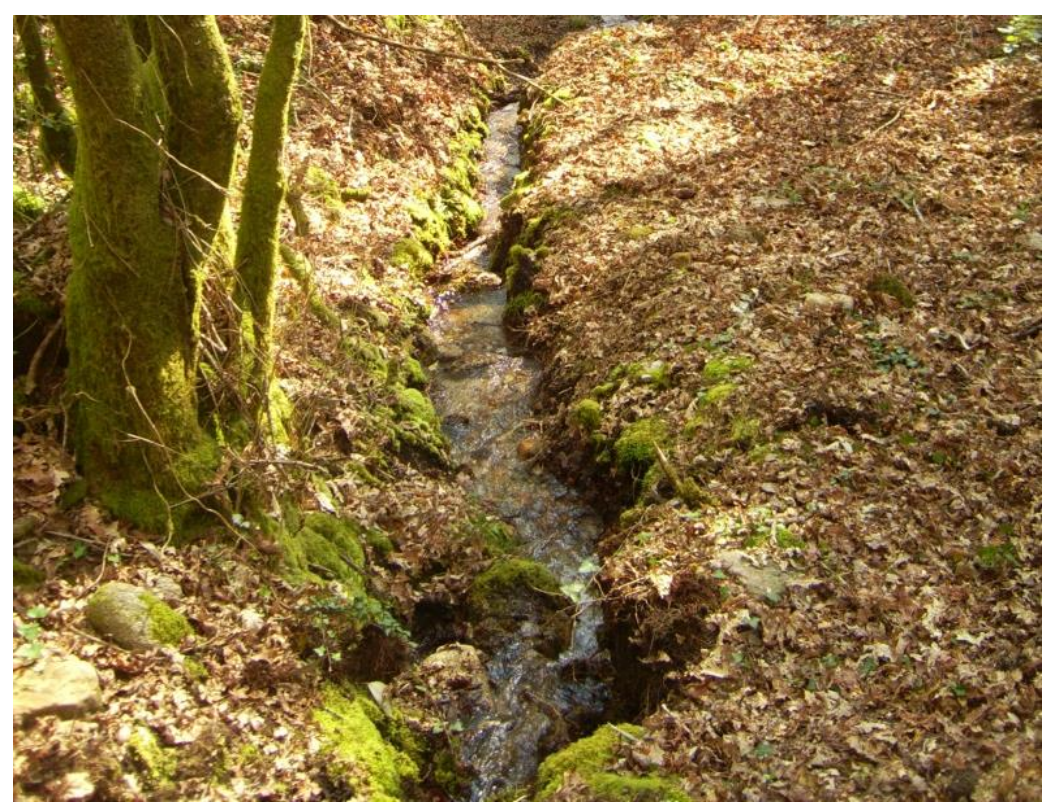

Fig. 4. Ejemplo de un rego tradicional, atravesando un robledal.

\section{De las pozas y presas a las fincas y prados a través de los regos}

No es mucho lo que se puede decir del modo en que se llevaba el agua a las diversas fincas y prados, ya que el Sistema es bastante simple. Desde la presa se construyeron, de la mano de los campesinos, unos regos que se van dividiendo y van llegando a cada una de las fincas, de diferente hondura dependiendo de si eran regos principales o secundarios. El propio lecho del riachuelo era utilizado como un rego más, utilizándolo ya para regar las fincas por las que pasa sin necesidad de crear otro paralelo, lo cual sería ilógico. Estos canales se van dividiendo entre si progresivamente formando un sistema que abarca amplias zonas del terreno, y que llegaban a cada una de las leiras y prados, teniendo muchas veces una longitud considerable.

A la hora de regar, el campesino al que le toque el agua, va tapando aquellos riegos por los que no quiere que pase el agua, guiándola hasta su finca. $\mathrm{Al}$ ser de pequeñas dimensiones y al no ser un caudal fuerte, este tapado se hace con piedras, terrones y barro.

Por otra parte, debemos hacer aquí mención de un mecanismo esencial de estos regos: el candoeiro, del que hablamos anteriormente. Se trata de un mecanismo utilizado por los agricultores con razón de dos ocasiones: por un lado, en aquellas presas, como ya vimos anteriormente, que no se encuentran en el propio lecho del riachuelo, y donde los campesinos tenían que cortar el curso natural de este guiando el agua por un rego construido ex profeso; y por otro lado, en aquellas presas que si se encontraban en el propio lecho, como punto intermedio entre las diferentes construcciones de este tipo existentes a lo largo del riachuelo.

Veamos en que consiste este mecanismo. Su función básica es la de guiar el agua de un rego principal para otro, y viceversa, desviar el agua de un rego para otro (normalmente uno de ellos es el lecho natural del riachuelo). Cuando la presa no está en el lecho, en la época de regadío, se corta el agua del lecho natural y se guía por un canal construido ex profeso (entre otras razones, a veces, por el escarpado del terreno que a veces impidió hacer una construcción de este tipo en el propio lecho); la explicación de la otra posibilidad se relaciona con la regulación conjunta de las aguas de las presas que están una a continuación de otra en un riachuelo. El agua de la primera presa baja por los regos a las fincas, 
y en un momento determinado, este se tiene que dividir en dos, uno que sigue para las fincas, y otro, que continúa hacia la siguiente construcción, siendo normal que este último sea el curso natural del riachuelo. En esta separación de los regos surge como solución este mecanismo.

Su estructura es simple. Se trata de una especie de pequeñas compuertas de madera, que impiden el paso del agua, y que por la parte en la que esta choca contra ellas, se hecha barro, piedras de poco tamaño y especialmente terrones. Al hecho de construir esta infraestructura se le llama facelo candoeiro y al hecho de cambiarlo de sitio, desviando el agua, botar abaixo o candoeiro.

\section{De la industrialización y los cambios morfológico-estructurales en el sistema de pozas y presas}

A partir de mediados de la segunda mitad del siglo XX Galicia sufrió una acelerada industrialización tardía que afectó y sigue afectando a la arquitectura popular de nuestro país con una inusitada fuerza. Este es un tema sobre el que han incidido etnógrafos y estudiosos en general ${ }^{4}$, quienes se han hecho eco de la problemática que esto supone en tanto en cuanto debido a esta apresurada industrialización "se están dando una serie de transformaciones, sustituciones y situaciones no estudiadas ni apropiadas" 5 que afectan sobremanera a este patrimonio etnográfico popular prácticamente infravalorado. Pocas son las construcciones de la arquitectura popular que se mantienen en pie tal y como eran en época preindustrial, sin transformaciones propias de esta nueva era en la que el ladrillo y el cemento son amos y señores y donde los elementos tradicionales quedan totalmente relegados.

Las construcciones tradicionales destinadas a la acumulación de agua sufrieron el envite de esta nueva época, siendo las presas las construcciones en donde más actuó la industrialización y donde más se dejan notar los cambios producidos, ya no sólo cambios en su morfología y estructura -en aras de un pensamiento de modernización y progreso-, sino en general en el modelo socioeconómico del rural gallego, donde el campo se ha ido paulatinamente olvidando y abandonando, provocando que a la par se haya producido lo mismo con algunas pozas y presas, en tanto en cuanto si las fincas no se cultivan, tampoco son necesarias algunas de estas construcciones, puesto que ya no hay campos que se vayan a regar.

El nuevo panorama socioeconómico, donde primaban otros sectores frente a la tradicional agricultura, supuso una estocada importante al antiquísimo y tradicional sistema de pozas y presas. Muchas de estas construcciones vieron su desaparición, debido al abandono de fincas y/o al cambio de su utilidad, ahora convertidas en montes. En estos casos, las construcciones de regadío fueron paulatinamente cubriéndose de maleza y de sedimentos, y a falta de limpieza y del cíclico acto de conservación de las mismas, fueron integrándose con su contorno, hasta tal punto que ahora ya es difícil discernir cual era su morfología y sus características originales. Otras veces, el panorama socioeconómico rural condujo a la canalización de ciertos riachuelos, convertidos en traídas de agua vecinales, provocando la total destrucción de esas centenarias construcciones populares.

\footnotetext{
${ }^{4}$ De Llano Cabado, P. (1981): Arquitectura popular en Galicia: a casa-vivenda, as serras, Santiago de Compostela, Publicacións do Colexio Oficial de Arquitectos de Galicia, pp. 137-139; Bas López, B. (1983): As construcións populares: un tema de etnografía en Galicia, Sada, Ediciós do Castro, pp. 252-263; Caamaño Suárez, M. (2003): As construccións da arquitectura..., op. cit., pp. 517-521.

${ }_{5}^{5}$ Bas López, B. (1983): As construcións populares..., op. cit., p. 252.
} 
Aun así, otras muchas pozas y presas siguieron siendo utilizadas, en tanto en cuanto Galicia y el rural siguió dedicando buena parte de sus esfuerzos a la agricultura, si bien con una importancia restringida. Esto supuso que muchas construcciones siguiesen en estado vivo, con uso, pero no supuso que permaneciesen con las características originales que poseían, sino que progresivamente se fueron introduciendo en ellas cambios trascendentales, notorios, en aras de un supuesto progreso y una modernización que aumentase la funcionalidad de las mismas.

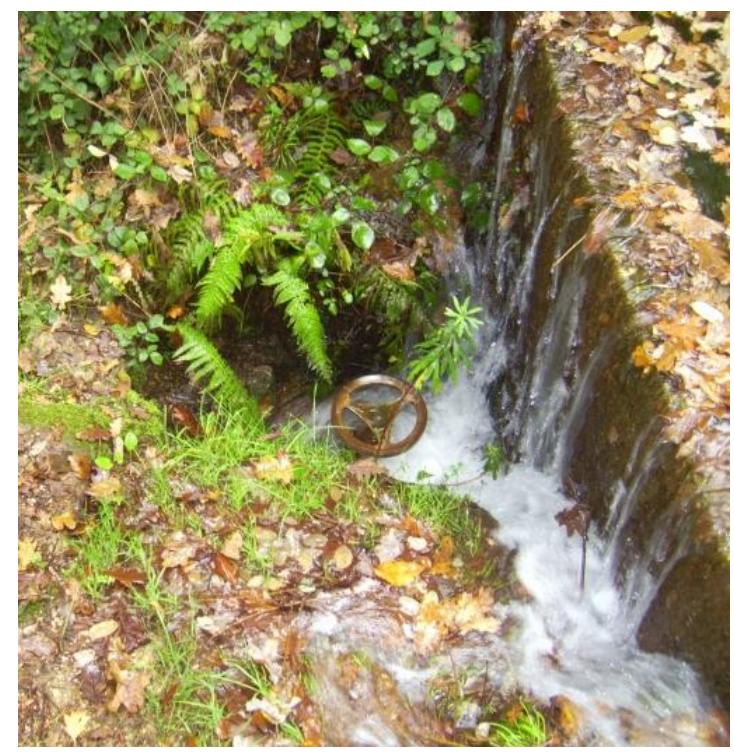

Fig. 5. La industrialización tardía supuso la transformación y modificación de algunas partes de las construcciones tradicionales de regadío. En la imagen, muro de la base de una presa con mecanismo moderno de apertura y cierre, donde antes estaba el buceiro.

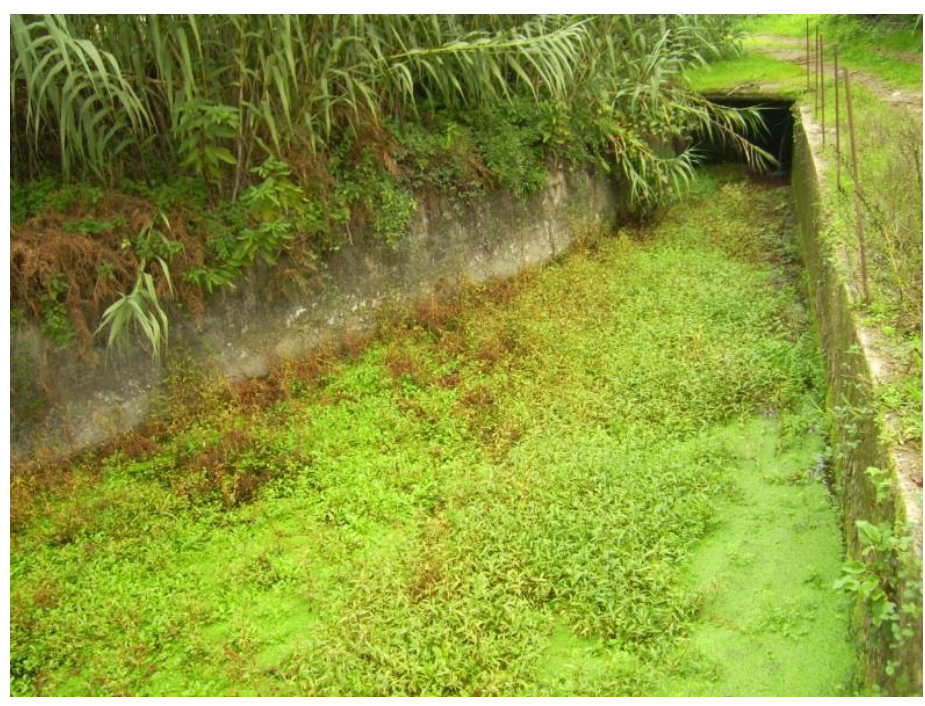

Fig. 6. Presa modificada enormemente, con muros actuales de hormigón y una parte de la misma soterrada bajo el camino lindante.

El cambio más importante vino de la mano del hormigón. La búsqueda de la máxima funcionalidad vio en este material moderno una forma de definir y fortalecer las paredes tradicionales de tierra o de piedras, que fueron substituidas en un alto porcentaje de pozas y 
presas, creándose en su lugar nuevas paredes que este nuevo material. Este cambio no sólo afectó a la estructura de la construcción, sino que produjo también un cambio en las tradiciones populares de las gentes del rural, puesto que la anual reunión de regantes para la limpieza, donde la maleza y tierra procedente del interior de la poza o presa ya no se necesitaba para su reutilización en la cíclica conformación de las paredes de las mismas, quedando todo este material amontonado al lado de estas construcciones sin utilidad alguna, afeando la construcción y mostrándose como lo que puede ser símbolo de los nuevos tiempos.

Otro cambio producido, acorde con la mentalidad utilitarista, de maximización de la funcionalidad y minimización del esfuerzo, fue la aparición de las máquinas excavadoras para la limpieza sobre todo de las presas de mayor envergadura, perdiéndose la tradicional costumbre vecinal de su limpieza en comunidad.

Por otra parte, si las paredes fueron modificadas, no iba a ser menos el mecanismo de salida del agua. Las diferentes estructuras pétreas, el boceiro, fueron progresivamente siendo sustituidas por mecanismos mecánicos modernos, metálicos. Esto supuso que las magníficas obras de arte de las pedras do buceiro fuesen rotas u olvidadas y enterradas, o en el mejor de los casos, posadas en algún lado de la presa, como muestra de lo que en su día fue, como una reminiscencia del pasado que se queda ahí para recordarnos que estamos en una nueva época donde todo está cambiando. Estas piedras fueron substituidas por planchas metálicas que se abren o se cierran con mecanismos actuales metálicos, mucho más prácticos que el tradicional sistema, más pesado, menos eficaz y más rudimentario. Actualmente, estas obras de arte labradas ya no se pueden encontrar, habiendo desaparecido con un poco de la historia de cada parroquia.

Los canales que salían de las pozas y presas también sufrieron el envite de los nuevos materiales, aunque en menor medida y menor importancia. Una parte de ellos, bastante pequeña (debido a la inmensidad de quilómetros y diversificaciones que éstos poseen por todo el territorio de una parroquia), fueron rehechos de nuevo con hormigón, con la finalidad de aprovechar al máximo el agua y que esta no se perdiese por ningún sitio. Casi siempre, por no decir siempre, se respectaron los recorridos por los que iban los canales tradicionales. Por su parte, los candoeiros apenas sufrieron de la mano de esta industrialización, debido sobre todo a su menos importante infraestructura y a su ubicación en lugares no centrales, lo que hizo que la mayoría se mantengan según el mecanismo tradicional. Otro aspecto del que debemos hacer mención son los cambios morfológicos y estructurales a que se vieron sometidas estas construcciones tradicionales cuando se encontraban al lado de algún ca-mino o carretera. La especial atención que recibieron las vías de comunicación en los últi-mos años del siglo XX y comienzos del XXI se tradujo en el hecho de que gran parte de los antiguos caminos se transformaron en carreteras, y esto supuso, en numerosas ocasio-nes, que se ampliase la anchura de las mismas para facilitar el tráfico de vehículos. Esta ampliación se hizo en muchos casos a costa del espacio que ocupaban las pozas y presas tradicionales en sus orígenes, modificándose su morfología tradicional, construyendo parte de la nueva carretera encima de la construcción tradicional, quedando el hueco necesario bajo la carretera para acumular agua, sin atender ni prestar atención a su morfología tradi-cional. En otras ocasiones, se produjo un traslado completo de la construcción a un lugar cercano. Esto supuso hacer de nuevo la construcción, destruyéndose la original y siendo la segunda una construcción nueva sin las características tradicionales de este tipo de cons-trucciones populares. 


\section{De la regulación del agua y de cómo los campesinos se dividían las horas y los días para regar}

Salvo los pozos, que eran normalmente de uso particular, tanto las levadas como las pozas y presas tenían un uso colectivo, siendo construidas y estando bajo la titularidad del conjunto de vecinos regantes. Esto hizo que los campesinos se tuviesen que poner de acuerdo entre sí para repartirse las horas y los días del agua, lo que trajo a lo largo de la historia constantes conflictos entre ellos ${ }^{6}$.

Antes de nada, debemos hacer varias puntualizaciones iniciales. Primeramente, debemos señalar que el sistema de repartición de las aguas es muy variado en Galicia, y existían numerosas formas de marcar los tiempos que le tocaba a uno u otro regante. Por otra parte, no debemos dejar de hacer referencia al hecho de que las aguas no pertenecen a las fincas, no es algo intrínseco a ellas, sino que, aunque están ligadas a éstas, eran consideradas como un bien más, privado, que se podía regalar, ceder o vender, pudiendo negociar con el separadamente de la finca ${ }^{7}$ y por último, debemos señalar que estos sistemas se prolongaban en el tiempo por medio y a través del derecho consuetudinario, es decir, las los tiempos de regar de cada campesino iban traspasándose oralmente de padres a hijos. Esto no implica que esta regulación del agua no fuese puesta por escrito, que también se llevó a cabo, pero como algo complementario al derecho consuetudinario; y por último, debemos tener en cuenta que los diversos sistemas de repartición varían en función de la cantidad de agua disponible y de la cantidad de fincas que tenían derecho al agua. No era lo mismo regar a través de una levada ${ }^{8}$ (aunque aquí entraba la problemática de si le robaban o no agua a los molinos, que dio también numerosos conflictos a lo largo de la historia), donde el caudal era siempre abundante y constante, como regar de una pequeña poza o de una presa para una multitud de fincas y prados.

Las pozas y presas se caracterizaban, sobre todo las últimas, en presentar un montón de campos y prados a los que tenían que llevar el agua, entrando aquí la característica gallega del minifundio. Los canales de regadío tenían a veces más de un quilómetro desde la construcción de acumulación de agua hasta la finca más alejada.

De aquí parte la complejidad de la regulación de los tiempos de regadío de cada uno de los campesinos con derecho a agua, surgiendo diversos sistemas, basados todos ellos en algún tipo de sistema de turnos, donde a cada finca le corresponde un tiempo determinado, que no es igual para todas las fincas, sino que varía muchísimo, con unas causas que se pierden en el olvido, pero que están en relación con las continuas luchas por apropiarse de este bien, por parte de los campesinos, que tantos conflictos generó, donde los intereses, sobre todo del clero y de la hidalguía, los llevaban a apropiarse de las aguas de otros vecinos, gracias al mayor poder social y económico que poseían, siendo muy cotidianas las denuncias, por ejemplo, a los curas, por pretender éstos tener más tiempos y llevar más agua a las tierras

\footnotetext{
${ }^{6}$ Candal González, X. M. (1993): Pleitos de Aguas en la Audiencia Coruñesa durante el siglo XVIII, Obradoiro de Historia Moderna, 2:85-103; Pérez García, J. M. (2003): Irriguer ou non? La guerre de l'eau en Galice (1600-1850), Histoire \& Societés Rurales, 20:37-52; Rey Castelao, O. (2012): La lucha por el agua en el país de la lluvia (Galicia, siglos XVI-XIX), Vinculos de Historia, 1:45-72.

${ }^{7}$ Rey Castelao, O. (2012): La lucha por el agua..., op. cit., p. 48.

${ }^{8}$ Las lovadas y su regulación se caracterizaba por tener un caudal importante de agua durante toda la época de regadío, siendo éste el motivo esencial que propició la aparición de un sistema específico para este tipo de construcciones. Este sistema es conocido comúnmente con el nombre de rego tumbado. En él, las fincas eran regadas por orden, una detrás de otra, cuando el campesino terminaba de regar la suya, comenzaba la del siguiente, hasta llegar al final, y volvía comenzar otra vez el ciclo. En este caso daba igual el tiempo que tardase el regante, puesto que la existencia de abundante agua y de forma constante permitía que todos pudiesen regar y que no sucediese que unos regaban con mucha agua y los siguientes no tuviesen ninguna.
} 
de sus casas rectorales o de su Iglesario"; del mismo modo, no debemos olvidar que este era un bien privativo que se podía regalar, ceder o vender, con lo cual a lo largo del tiempo esto afectó también a que los tiempos de agua de una finca a otra varíen mucho entre sí. Así, el hecho de que una finca esté cerca de una poza o presa y que la facilidad de hacer llegar el agua sea alta, no implica que estas tengan derecho sobre ella, pudiendo ser de secano.

Este sistema de turnos también varía en función de la propia poza o presa, del caudal que esta tenga y del número de fincas a regar. Algunas veces, la gran cantidad de agua y/o el pequeño número de fincas con derecho al agua hizo que algunas pozas y presas se rieguen solo de día, mientras que otras exprimen el tiempo tanto que riegan de noche, domingos y festivos, cuando son muchos los regantes y muy poca la cantidad de agua.

Otras veces surgían otras soluciones y sistemas, como el sistema de la corredía. Este sistema implicaba que cada regante tenía un tiempo determinado para regar que se controlaba de la siguiente manera: se tiraba en la poza o en la presa un higo (en tiempos más recientes, se cambió por otras cosas, como bombillas) y el campesino podía regar el tiempo que tardase éste en llegar a su finca, que normalmente era de varias horas. Cuando llegaba, comenzaba a regar otro, volviendo a tirar otro higo.

Por su parte, en relación con la mayor o menor necesidad de regadío, las regas se iniciaban en días diferentes, dependiendo de las tradiciones locales de cada parroquia, lugar, e incluso de la propia tradición de cada poza y de cada presa. Normalmente se comenzaba a regar con San Juan (24 de junio) o San Pedro (29 de junio), y se regaba entre veinte días y dos meses, aproximadamente, dependiendo de cada lugar, si bien cada poza o presa se inicia siempre y cada año el mismo día, rematando también en el mismo cada año, estando estipulado este según el derecho consuetudinario.

Para terminar este apartado sobre la regulación del agua, debemos hacer mención de una práctica importante, que mencionamos anteriormente: el derecho escrito. Como ya dijimos anteriormente, es el derecho consuetudinario el que domina y gobierna todo el derecho de regadío en Galicia. Las gentes del rural van aprendiendo y pasando, de forma oral, esta regulación a las nuevas generaciones. Sin embargo, este derecho consuetudinario fue a veces también escrito, y aunque no se puede determinar a ciencia cierta, ya que el número de pozas y presas que aún se mantienen en uso es enorme, y mayor sería en tiempos pasados antes de la actual desaparición de muchas, podemos afirmar que para la gran mayoría se crearon documentos de regulación y de los turnos que le tocaban a cada regante en el siglo XVIII. Estos documentos son conocidos con el nombre de prorrateos, prorreteos o prorroteos. Su importancia es tal que merecerían un estudio específico.

Dado la época de la que estamos hablando, debemos precisar que sus autores serían las élites locales de cada parroquia, fundamentalmente los curas y los hidalgos, ya que el $99 \%$ de los campesinos no sabrían ni leer ni escribir (en consonancia con lo dicho anteriormente de que estos curas e hidalgos eran los litigantes de los pleitos por agua más comunes). Estos lo verían como un medio de legitimar el derecho consuetudinario, para que hubiese menos luchas entre los vecinos y sobre todo, para reafirmar, seguramente, una regulación muy favorable a sí mismos y a sus tierras.

Estos documentos ofrecen al historiador local una información muy valiosa sobre diversos aspectos culturales, económicos, etc. Sin embargo, encontrarlos actualmente es muy difícil,

\footnotetext{
${ }^{9}$ Rey Castelao, O. (2012): La lucha por el agua..., op. cit., pp. 61-64.
} 
por diversos motivos. El primero de ellos es que, en una época en la que casi nadie sabía leer y escribir, solo eran guardados por una o dos personas en la parroquia, los que los hicieron, lo que implicó que con el paso del tiempo muchos se perdiesen. Por otra parte, estos prorrateos, aquellos que fueron sobreviviendo y que tuvieron la suerte, en algún momento, de ser copiados, fueron, en muchos casos, eliminados con conocimiento de causa, como método para hacerse con las aguas de las fincas abandonadas o de otra gente, para eliminar las evidencias de que los derechos usurpados no les pertenecían realmente. Este hecho progresivo llevó a que numerosos campos y prados gocen actualmente de tiempos de aguas mayores de los que realmente tenían, o que otras que ni siquiera tenían derecho, por cualquier razón, se rieguen ahora. Esto es problemático sobre todo en el siglo XX, que con la industrialización y el paulatino abandono del campo, algunos vecinos vieron en este modus operandi un medio para obtener más agua para sus fincas, eliminando las pruebas y las evidencias documentales que les estorbaban, con el fin de que ningún vecino le pudiese reclamar los nuevos derechos que 'adquirían' -usurpaban- de otras fincas y prados abandonados.

\section{Conclusiones}

El sistema de regadío tradicional es en el mundo rural una de las facetas más interesantes e importantes de la comunidad aun actualmente, pero su estudio no ha gozado de las simpatías ni del interés de la comunidad científica, ni siquiera de los etnógrafos, que salvo algún estudio aislado o alguna mención muy específica, apenas se han parado a estudiar esta temática.

El análisis y estudio de los sistemas de pozas y presas no debe ser entendido tan sólo en tanto en cuanto construcciones destinadas a la acumulación de agua, desde una óptica funcional, sino que su mayor importancia radica especialmente en el carácter colectivo que este tipo de construcciones tienen, que potencian el sentimiento de unión de la comunidad y también, al mismo tiempo, sentimientos de desunión y reyertas entre los vecinos, dando como resultado final que alrededor de las pozas y las presas y a través de su cuidado y de las diversas tareas que se hacen en comunidad a su alrededor, como la de la limpieza de las mismas, provocan que dichas construcciones posean una dimensión además de funcional y de georreferenciadores territoriales, una dimensión social entre los vecinos, que es de las más importantes.

Esta dimensión social, sin embargo, está desapareciendo a pasos agigantados debido a los cambios de vida actual, olvidándose esa parte por parte de los campesinos, viéndolas solamente en tanto en cuento su función funcional. Ello ha traído, como hemos visto, además de los numerosos cambios físicos, también cambios en las costumbres sociales, desapareciendo paulatinamente las diferentes tareas que se hacían en comunidad, siendo todo ello suplantado por el individualismo propio de la globalización.

Por ello, se debe insistir y recuperar en estas tradiciones, y se debe dar a conocer, mediante estudios científicos, la realidad tan importante que estas construcciones tradicionales tuvieron a lo largo del tiempo. Esto que aquí decimos, es válido también para otro tipo de arquitectura tradicional como las fuentes, los lavadores tradicionales, los molinos, etc., que tienen la misma problemática y que paulatinamente están desapareciendo. 

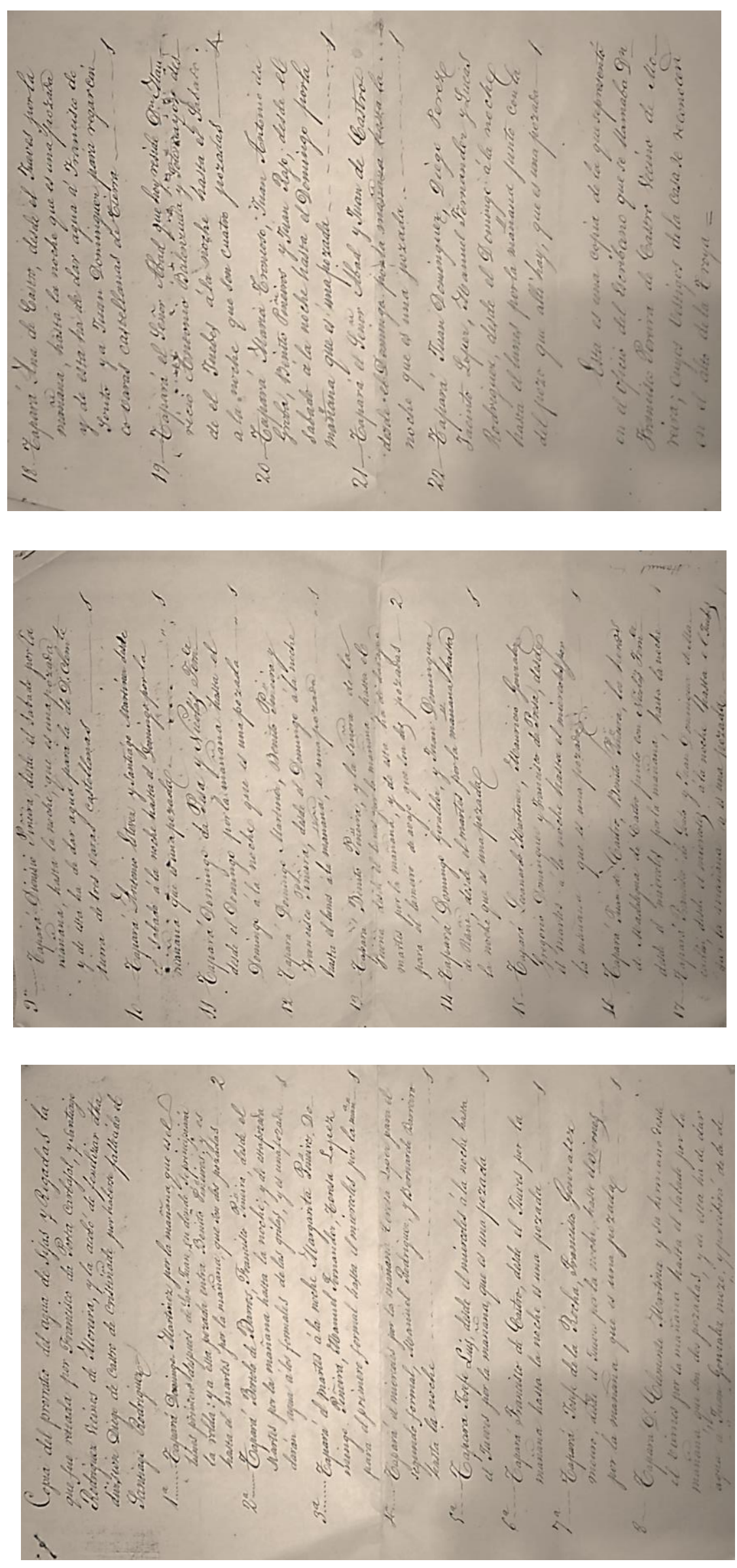
Fig. 7. (Página antetior) Prorrateo de una presa da parroquia de Moreira (Ponteareas, Pontevedra). El documento existente actualmente es una fotocopia de una fotocopia de una copia (como se dice al final del documento) del supuesto prorrateo original. Se ve, pues, las dificultades que su conservación hasta nuestros días han tenido este tipo de documentos, razón por la cual la mayoría de los mismos han desaparecido hoy en día.

\section{Referencias}

Bas López, B. (1983): As construcións populares: un tema de etnografía en Galicia, Sada, Ediciós do Castro.

Caamaño Suárez, M. (2003): As construccións da arquitectura popular: Patrimonio etnográfico de Galicia, Santiago de Compostela, Consello Galego de Colexios de Aparelladores e Arquitectos Técnicos.

Candal González, X. M. (1993): Pleitos de Aguas en la Audiencia Coruñesa durante el siglo XVIII, Obradoiro de Historia Moderna, 2:85-103.

De Llano Cabado, P. (1981): Arquitectura popular en Galicia: a casa-vivenda, as serras, Santiago de Compostela, Publicacións do Colexio Oficial de Arquitectos de Galicia.

Pérez García, J. M. (2003): Irriguer ou non? La guerre de l'eau en Galice (1600-1850), Histoire \& Societés Rurales, 20:37-52.

Rey Castelao, O. (2012): La lucha por el agua en el país de la lluvia (Galicia, siglos XVIXIX), Vinculos de Historia, 1:45-72.

Vázquez Martínez, A. (1946-1947): Las 'levadas’ de Arbo, El Museo de Pontevedra, 4:172185. 